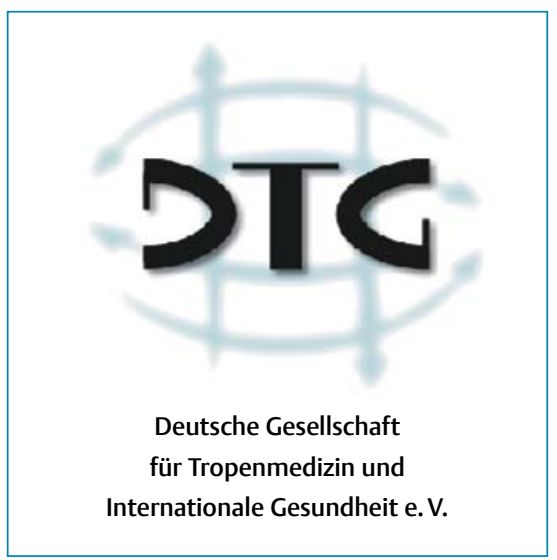

\title{
Liebe Mitglieder und Freunde der DTG,
}

inzwischen liegt der Kopenhagener Europäische Tropenmedizinkongress hinter uns, einen Bericht von unserem Vorsitzenden Thomas Löscher finden Sie auf der nächsten Seite. Mit dieser Veranstaltung ist nun auch Grönland offiziell zu einem tropischen Land aufgestiegen. Als unseren Delegierten in die Federation of European Societies for Tropical Medicine and International Health (die FESMIH) haben wir wieder Thomas Junghanss für einen Zeitraum von 2 Jahren entsandt.

Vor einigen Wochen haben wir hier in Hamburg unseren Freund, Kollegen und ehemaligen Vorsitzenden der DTG, Professor Gerd Burchard in den Ruhestand verabschiedet - kaum vorstellbar, dass die Tropenmedizin in Hamburg nun ohne ihn weitergehen soll - und ganz wird es auch nicht so sein. Mit seiner ruhigen und überlegten Art und seinem enzyklopädischen tropenmedizinischen Wissen ist er für viele Tropenmediziner und Ärzte meiner Generation und die Jüngeren ein wichtiges Vorbild und begeisternder Lehrer geworden.
Aktuelles zu Masern- und Gelbfieberimpfstoffen

Zur Zeit sind die Masern ja wieder auf dem Vormarsch - in Deutschland sind schon jetzt 10-mal soviel Fälle zu verzeichnen wie im letzten Jahr (!), was für uns alle Anlass sein sollte, auch bei der reisemedizinischen Beratung verstärkt auf den Masernimpfschutz zu achten. Zur Sicherheit von Masernimpfstoffen hat das PEI mit dem Bundesinstitut für Arzneimittelsicherheit in seinem neuen Bulletin Informationen dargelegt: www.pei.de/ bulletin-sicherheit.

$\mathrm{Da}$ es voraussichtlich in den nächsten Wochen zu einer Knappheit auch bei der Verfügbarkeit bei Gelbfieberimpfstoff kommen wird, haben die Deutsche Fachgesellschaft für Reisemedizin und die DTG im Einvernehmen mit dem PEI und dem Hersteller Sanofi Pasteur eine Stellungnahme zu Produktionsproblemen und möglichen Versorgungsengpässen beim Gelbfieberimpfstoff Stamaril ${ }^{\circledR}$ verfasst, veröffentlicht in dieser Ausgabe, S. 217, und im Internet: www.dtg.org.

\section{Interessantes, informatives und umfangreiches Pi}

Vom 10. bis 13. September fand in Kopenhagen der $8^{\text {th }}$ European Congress on Tropical Medicine and International Health (ECTMIH) statt. Die seit 1995 meist im Zweijahresrhythmus abgehaltene Konferenz der aus 15 nationalen Fachgesellschaften bestehenden FESTMIH (Federation of der European Societies for Tropical Medicine and International Health) wurde diesmal von der Dänischen Gesellschaft für Tropenmedizin und Internationale Gesundheit im übersichtlichen und stadtnahen Tivoli Congress Center ausgerichtet.

Umfangreiches Programm und breites Themenspektrum

In 4 zum Teil als Podiumsdiskussion organisierten Plenarsitzungen und in 71 Symposien wurden 120 eingeladene Vorträge und 60 wissenschaftliche Kurzvorträge gehalten. Zudem gab es 10 frühmorgend- liche Meet-the-expert-Sessions, mehrere Workshops und mehr als 300 wissenschaftliche Poster. Gleichzeitig fand an 2 Tagen die $5^{\text {th }}$ Conference of the Scandinavian-Baltic Society for Parasitology statt, deren 13 Symposien und Workshops von allen Kongressteilnehmern ohne zusätzliche Kosten besucht werden konnten. Insgesamt ergab sich somit die Qual der Wahl aus 6 bis 7 Parallelsitzungen. Trotz dieses sehr umfangreichen Programms waren die Symposien übersichtlich und sinnvoll in 6 Tracks organisiert, die die Auswahl erleichterten (infectious diseases, reproductive \& child health, non-communicable diseases, health systems \& resources, global interaction \& health, parasitology).

Das Schwerpunktthema waren die Millenium Deveplopment Goals:

- Was wurde bisher erreicht und was nicht? 
Termine

Aufmerksam machen möchten wir Sie gern noch einmal auf das Malariameeting, das vom 8. bis 9. November 2013 in der RWTH in Aachen stattfindet. Einzelheiten darüber finden Sie auf der Homepage der DTG oder unter www.malaria-aachen. de/.

Weitere interessante Termine sind:

- die Jahrestagung der Gesellschaft für Tropenpädiatrie vom 31. Januar bis 2. Februar 2014 in Würzburg, www. tropenpaediatrie.de

- eine schifffahrtsmedizinische Veranstaltung mit dem Titel „Interkulturelle Kompetenz an Bord" am 14. und 15. Februar 2014 in Hamburg, www. schiffsarztlehrgang.de/sonderveran staltungen.html, unter anderem mit Referaten zu den Themen „Seefahrerkulturen in Ozeanien“ und „Den Fremden gibt es nicht - häufige Trugschlüsse im Umgang mit anderen Kulturen"

- die Fachmesse „Engagement weltweit" zur Arbeit von Fachkräften in Entwicklungsländern am 16. November 2013 in Bonn, veranstaltet vom Arbeitskreis „Lernen und Helfen in Übersee“ und unterstützt vom BMZ
Preis für Tropenmedizin

Weiterhin ist der Preis für Tropenmedizin der DTG ja ausgeschrieben, denken Sie daran, dass die Bewerbungs- und Vorschlagsfrist dafür am 31. Dezember 2013 abläuft.

\section{Vorstandswahlen}

Leider haben wir bisher noch fast keine Vorschläge $\mathrm{zu}$ den anstehenden Vorstandswahlen und zur Wahl eines neuen Schriftführers erhalten - bitte lassen Sie uns diese doch in den nächsten Wochen zukommen - ein Mitglied schlug allerdings originell vor, doch mal einen „Mäuseprofessor“ und nicht wie gewohnt einen Kliniker zum 1. Vorsitzenden zu wählen. Neben der anstehenden Wahl wird auf der Vorstandssitzung am 16. Oktober 2013, die schon gelaufen sein wird, wenn Sie dies lesen, die Vorbereitung auf unseren Kongress in Düsseldorf im März des kommenden Jahres das Hauptthema sein.

Mitgliedsbeiträge zweimal eingezogen Leider hat unsere Bank ohne unsere Mitwirkung von zahlreichen Mitgliedern, die ein Abonnement der Zeitschrift TM\&IH haben, am 22. und am 24. Juli 2013 den Beitrag für die DTG und für das
Abo, entsprechend 130 Euro, zweimal abgebucht. Wir bedauern dies sehr und auch die Bank hat sich nachdrücklich dafür entschuldigt. Sie müssen, wenn der Fehler bei Ihnen bisher noch unentdeckt geblieben ist - was eigentlich unwahrscheinlich ist, da wir alle Betroffenen angeschrieben haben - sich an Ihre eigene Bank wenden und „Rückgabe wegen Widerspruchs aufgrund doppelten Einzugs“ verlangen.

Mit herzlichen Grüßen aus München und Hamburg

Thomas Löscher und Hinrich Sudeck
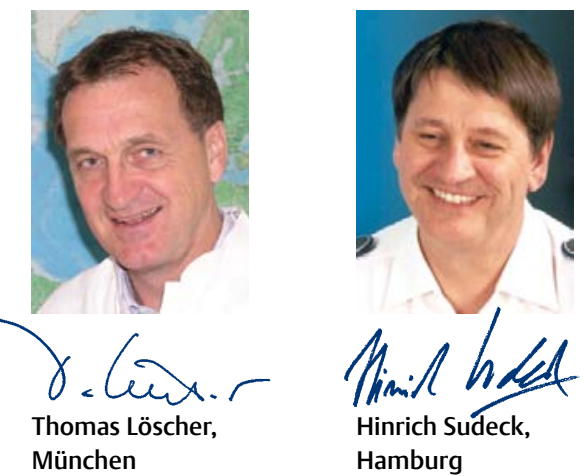

München
Süden entwickelte sich eine intensive und sehr fruchtbare Diskussion über Erfolge, Defizite, Finanzierung und Notwendigkeit der Forschung der armen Länder zu ihren eigenen Gesundheitsproblemen sowie über die Probleme mit denen die Forscher dort konfrontiert sind.

breites Spektrum weiterer Themen von globaler Gesundheit über Infektionskrankheiten und nicht übertragbare Krankheiten bis zu Psychiatrie, Arbeitsmedizin und Palliativmedizin in Ländern mit mangelnden Ressourcen. Als sehr interessant erwies sich auch ein Symposium mit besonderem Lokalkolorit über die Gesundheitsprobleme in Grönland.

\section{Gut besuchtes DTG-Symposium}

Die DTG veranstaltete ein gut besuchtes Doppelsymposium zum Thema ,NorthSouth research partnership'. Am Beispiel verschiedener Forschungskooperationen deutscher Einrichtungen mit Partnern im noch eine Posterbegehung, bei der die Autoren ihre Ergebnisse vorstellen konnten. Ein Verzeichnis von Postern und Posterautoren war nur im elektronischen Abstractbook zu finden, einer nur online verfügbaren Sonderausgabe der Zeitschrift Tropical Medicine \& International Health. Gerade für Nachwuchswissenschaftler sowohl in unseren Partnerländern im Süden wie auch in Europa erhöht dies nicht unbedingt die Motivation an ECTMIHKongressen teilzunehmen. Es ist zu hoffen, dass dies bei den zukünftigen ECTMIHs besser gehandhabt wird. Auch bei den 2014 anstehenden Tagungen, die die DTG ausrichtet oder bei denen sie beteiligt ist, sollte dies unbedingt berücksichtigt werden. Der nächste European Congress on Tropical Medicine and International Health ( $9^{\text {th }}$ ECTMIH) wird vom 6. bis 10. September 2015 in Basel stattfinden.

Prof. Dr. Thomas Löscher, München 